\title{
A Note on the Acceptability of Regression Solutions: Another Look at Computational Accuracy
}

\section{ANTONI ESPASA*}

This note examines the experiment performed by Beaton, Rubin, and Barone (1976) to study the effect of rounding errors in published figures, when these data are used in regression analysis. The experiment could be vitiated by the fact that the error introduced in the trend variable is by no means trivial when one measures the data in deviation from the mean. For this reason, the results presented in Beaton, Rubin, and Barone (1976) do not contain enough evidence to suggest "that it is extremely unlikely that the unperturbed solution" (p. 161) of the Longley model is the correct one.

KEY WORDS: Regression analysis; Rounding adjustments.

One of the aims of the article by Beaton, Rubin, and Barone (1976) is to investigate the effect of rounding adjustments in published figures on the regression estimates with these data. They take the data used in Longley (1967), generate random numbers between -.5 to $.49 \ldots$ and add them to the digit after the last published digit of the regressor series. They say "the error introduced by such rounding would seem to be trivial, since the data are presented with three to six digits of accuracy" (p. 60). But since the regressions include a constant term, we need to compare the amount of the rounding with the deviation from the mean of the series. ${ }^{1}$ On a priori grounds, the maximum amount of rounding should be trivial for the first five regressors $\left(X_{1}\right.$ to $\left.X_{5}\right)$, but not for the trend variable $X_{6}$, since .5 is 10.5 percent of the standard deviation of $X_{6}$. Therefore, the 1,000 experiments that they carried out on 1,000 plausible sets of the

* Antoni Espasa is research officer, Banco de España, Madrid, 14-Spain.

${ }^{1}$ We are assuming that the rounding adjustments are random and their mean is zero. six independent variables can differ by something more than trivial amounts.

The same can be said for the difference between their experiments and the regression with the data as published and estimated by highly accurate programs (the unperturbed solution). Therefore, it is not quite correct to conclude, on the basis of their experiment, that "it is extremely unlikely that the unperturbed solution is the 'correct' solution of this problem" (p. 161) because the problem in their experiment could differ more than trivially from the one considered in the unperturbed solution.

Since the question of whether highly accurate programs are able to solve regressions with highly correlated regressors is important, it would be interesting if the authors could rerun their experiment with the six regressors but perturb only the data for $X_{1}$ to $X_{5}$.

[Received June 1976. Revised January 1977.]

\section{REFERENCES}

Beaton, Albert E., Rubin, Donald B., and Barone, John L. (1976), "The Acceptability of Regression Solutions: Another Look at Computational Accuracy," Journal of the American Statistical Association, 71, 158-68.

Longley, James W. (1967), "An Appraisal of Least Squares Programs for the Electronic Computer from the Point of View of the User," Journal of the American Statistical Association, 62, 819-41.

(c) Journal of the American Statistical Association September 1977, Volume 72, Number 359 Theory and Methods Section 